\title{
Risk Factors Associated with Anthracycline Induced Cardiac Dysfunction in Pediatric Patients
}

\author{
Abdul Sattar Shaikh1, Muhammad Matloob Alam', Shazia Samad Mohsin², Qalab Abbas', \\ Zehra Fadoo', Mehnaz Atiq ${ }^{*}$ \\ ${ }^{1}$ Department of Paediatrics and Child Health, Aga Khan University Hospital, Karachi, Pakistan \\ ${ }^{2}$ Department of Pediatrics, Liaquat National Hospital and Medical College, Karachi, Pakistan \\ Email: * Mehnaz.atiq@aku.edu
}

Received 20 April 2014; revised 31 May 2014; accepted 18 June 2014

Copyright (C) 2014 by authors and Scientific Research Publishing Inc.

This work is licensed under the Creative Commons Attribution International License (CC BY). http://creativecommons.org/licenses/by/4.0/

c) (i) Open Access

\section{Abstract}

Anthracyclines (i.e., doxorubicin, daunorubicin) have significant impact on outcome in many pediatric chemotherapy protocols and therefore remain the mainstay of treatment. The objective of this study was to identify the risk factors for anthracycline induced cardiac dysfunction in pediatric patients. Multiple logistic regression model was applied to assess the risk factors for development of cardiac dysfunction. 110 pediatric oncology patients were available for final analysis. 75 (66\%) children were males and mean age was $74 \pm 44$ months. ALL $(n=70,64 \%)$ was the most common primary diagnosis followed by lymphoma $(n=19 ; 17 \%)$ and AML $(n=12,11 \%)$. Daunorubicin alone or in combination with doxorubicin was used in $(n=94,85 \%)$ patients and cumulative dose $<300 \mathrm{mg}$ was used in $(n=95 ; 86 \%)$ children. $24(22 \%)$ children received radiation therapy as per protocol and sepsis were observed in 47 (43\%) cases. Post anthracycline, 15 (14\%) children had cardiac dysfunction within a month; out of them 10/15 (67\%) had isolated diastolic dysfunction, while $28(25 \%)$ developed dysfunction within a year. $19(17 \%)$ had pericardial effusion. 11 expired and out of them, 7 had significant cardiac dysfunction. Cumulative dose $>300$ $\mathrm{mg} / \mathrm{m}^{2}(p<0.001 ;$ AOR 2.3$)$, radiation therapy $(p=0.009 ;$ AOR 3.5$)$ and sepsis $(p=0.002 ;$ AOR 2.6) were found to be independent risk factors associated anthracycline induced cardiac dysfunction. At univariant level use of daunorubicin alone or in combination therapy $(p<0.001,0 R 7)$ and mode of delivery ( $p$ 0.048, OR 9.7) were also found statistically significant. In conclusion anthracycline induced cardiac dysfunction is mostly related to cumulative dose $>300 \mathrm{mg} / \mathrm{m}^{2}$, use of Daunorubicin alone or in combination with doxorubicin, mode of delivery, radiation therapy and sepsis. Regular long term follow-up with cardiologist is the key point for early diagnosis and therapy for a long term survival.

\footnotetext{
*Corresponding author.
} 


\section{Keywords}

\section{Anthracycline, Cardiac Dysfunction, Risk Factors, Pediatric Oncology}

\section{Introduction}

The anthracycline and related compounds (doxorubicin, daunorubicin) are the backbone of current childhood chemotherapy (solid and hematological malignancies) [1]. Despite the fact that they are very potent and highly effective chemotherapeutic agents, their cardiotoxicity remains a serious concern during management [1] [2], with the incidence of cardiotoxicity ranges between 0 - 57 percent [3]. Their main adverse effects include systolic and diastolic dysfunction, cardiomyopathy, arrhythmias and pericardial effusion [1] [2] [4]. As the myocardial injury predicts the future development and severity of long term ventricular dysfunction, regular evaluation and management of early onset cardiac adverse events are important in management of children receiving anthracyclines [5].

Certain potential risk factors including age, sex, race, associated congenital or acquired heart disease, type, mode and cumulative anthracycline dosage, concomitant radiation therapy, use of other cardiotoxic or cardioprotective drugs, Down's syndrome, sepsis during management and type of malignancy have been evaluated in medical literature [1] [3] [6]-[11]. Therefore this is paramount to identify potential risk factors associated with increase cardiotoxicity so the initial damage to the heart can be prevented by adapting preventive strategies and early start of cardiac failure treatment if needed [12]-[15].

There is very little literature from the subcontinent on risk factors associated with anthracycline induced cardiotoxicity in pediatric oncology patients. The aim of this study was to identify the risk factors for cardiac dysfunction associated with anthracyclines in pediatric patients.

\section{Patients and Methods}

We performed a prospective cohort study during July 2010-June 2012 at the department of paediatric and child health, Aga Khan University, Hospital, Karachi, Pakistan. All pediatric oncology patients aged 0 to 16 years, who received anthracycline as a part of their chemotherapy protocol and remain in regular follow-up for at least 1 year post chemotherapy and had at least 3 echocardiographic studies within a year (at baseline, within 1 month and within a year) were included for final analysis. Children with any structural cardiac condition or cardiomyopathy were excluded. Data were collected on a structured proforma. Risk factors taken into consideration were the primary diagnosis, age, gender, type, mode of delivery and cumulative dose of anthracycline, radiation therapy, Down's syndrome and culture proven sepsis. Follow-up compliance was also recorded. Echocardiographic studies for functions (baseline, within a month and within a year post anthracycline exposure for acute and early onset chronic progressive cardiotoxicity respectively) were recorded. 190 children were enrolled in this study according to study criteria but 80 patients (42\%) were further excluded due to poor or no follow-up, treatment migration, self treatment withdrawal. Thus 110 children were available for final analysis.

\section{Data Collection and Analysis}

Statistical analysis was performed by using SPSS software package (version 20.0, SPSS). Results were presented as mean and standard deviation for continuous variables (i.e., age, height, weight, body surface area, cumulative dose, echocardiographic evaluation) and frequency and percentage for categorical variables (i.e., gender, type of malignancy, and type of Anthracycline). Risk factors for anthracycline induced cardiac dysfunction were identified by comparing the patients with and without cardiac dysfunction. A $p$-value of 0.05 was considered significant at the univariate level. For the multivariate model a $p$-value cut-off of 0.01 along with high crude odds ratio were taken into account. Finally, a multiple logistic regression model was applied for all variables in such a way that the variable with the most significant $p$-value was entered first in the final model to calculate the adjusted odds ratio.

\section{Echocardiographic Analysis}

By using Philips IE33 ((Philips Medical Systems, Andover, MA, USA) and GE vivid 7 Pro (General Electric 
Company, NYSE: GE, UK) echocardiographic machines. Real time images were obtained from standard projections. Transmitral flow velocity pattern was recorded from the apical four chamber view. M-mode was taken from parasternal long axis view. A baseline echocardiography was done in all patients as required in the management protocol. Echocardiographic assessment of systolic and diastolic left ventricular function and pericardial effusion was made. Shortening fraction (FS) and Ejection fraction (EF) calculated for systolic functions while trans-mitral flow velocities their ratio, Deceleration Time (DT) and Myocardial performance index (MPI or Tei index) [14]-[17] were evaluate for diastolic function at baseline, one month and 1 year post chemotherapy for acute and early onset chronic anthracycline cardiotoxicity.

\section{Results}

Of the 110 patients, $68 \%$ were males, mean age was $74 \pm 44$ months, and mean body surface area was $0.54 \pm$ $0.32 \mathrm{~m}^{2}$. Patients demographic features, risk factors and clinical profile mentioned in (Table 1).

Table 1. Demographic features of study population $(n=110)$.

\begin{tabular}{|c|c|}
\hline Demographic features & N (\%)/mean \pm SD \\
\hline Age & $74 \pm 44$ months \\
\hline Sex & \\
\hline Males & $75(68 \%)$ \\
\hline BSA & $0.84 \pm 0.32$ \\
\hline \multicolumn{2}{|l|}{ Type of malignancy } \\
\hline ALL & $70(64 \%)$ \\
\hline AML & $12(11 \%)$ \\
\hline Hodgkin + Non Hodgkin & $19(17 \%)$ \\
\hline Miscellaneous (Ewing Sarcoma = 4, Wilms tumor = 5). & $9(8 \%)$ \\
\hline \multicolumn{2}{|l|}{ Type of anthracycline used } \\
\hline - Doxorubicin & $59(53.5 \%)$ \\
\hline - Daunorubicin & $16(14.5 \%)$ \\
\hline - Both & $35(32 \%)$ \\
\hline \multicolumn{2}{|l|}{ Cumulative anthracycline dose } \\
\hline$<300 \mathrm{mg}$ & $95(86 \%)$ \\
\hline$>300 \mathrm{mg}$ & $15(14 \%)$ \\
\hline Radiation therapy & $24(22 \%)$ \\
\hline Down’s syndrome & $8(7 \%)$ \\
\hline Culture proven sepsis & 47 (43\%) \\
\hline Gram positive bacteria & $29(61.7 \%)$ \\
\hline Gram negative bacteria & $8(17 \%)$ \\
\hline Gram positive and negative combine & $6(12.8 \%)$ \\
\hline fungus & $4(8.5 \%)$ \\
\hline \multicolumn{2}{|l|}{ Cardiac dysfunction } \\
\hline - Within a month dysfunction & $15(14 \%)$ \\
\hline Diastolic alone & $10 / 15(67 \%)$ \\
\hline Combine systolic and diastolic & $5 / 15(33 \%)$ \\
\hline - Within a year dysfunction & $28(25.4 \%)$ \\
\hline Diastolic alone & $12 / 28(43 \%)$ \\
\hline Combine systolic and diastolic & 16/28 (57\%) \\
\hline \multicolumn{2}{|l|}{ Sign symptoms of cardiac failure } \\
\hline - Within a month & $4(3.6 \%)$ \\
\hline • Within a year & $18(16 \%)$ \\
\hline \multicolumn{2}{|l|}{ Pericardial effusion } \\
\hline - Within a month & $9(8.2 \%)$ \\
\hline - Within a year & 19 (17.1\%) \\
\hline
\end{tabular}


Most of the children had primary diagnosis of Acute Lymphoblastic lymphoma (ALL) (64\%) followed by Hodgkin and Non-Hodgkin lymphoma (17\%). Doxorubicin alone was used in 53.5\% of children and combination therapy in 32\%. Anthracycline cumulative doses were $<300 \mathrm{mg}$ in most of children 95 (86\%). Anthracycline was infused in 4 patients (3.6\%) while all others received boluses. Radiation therapy was used in 24 (22\%) children. None of the patient received any type of cardioprotective agents prophylactically. Eight children had trisomy 21.

Within a month echocardiography showed that fifteen (13.6\%) children developed cardiac dysfunction and most of them had isolated diastolic dysfunction 10/15 (66.6\%), while pericardial effusion was found in 9 patients. 28 (25\%) developed cardiac dysfunction when echocardiographic study was reviewed after a year, 12/28 (43\%) had isolated diastolic dysfunction and pericardial effusion was observed in 19 patients. Forty-seven (43\%) children admitted due to sepsis. eleven children expired, out of them 7 had significant cardiac dysfunction.

Multiple logistic regression analysis was performed for the identification of risk factors for anthracycline induced cardiac dysfunction and crude and adjusted Odds Ratio (AOR) was calculated (Table 2). The analyses did not signify age, sex, type of malignancy and Down's syndrome as risk factors. Cumulative dose $>300 \mathrm{mg} / \mathrm{m}^{2}$ ( $p$ $<0.001$; AOR 2.3), radiation therapy ( $p=0.009$; AOR 3.5) and sepsis $(p=0.002$; AOR 2.6) were found as independent risk factors associated anthracycline induced cardiac dysfunction in pediatric patients. At univariate level use of daunorubicin alone ( $p<0.001$, OR 7$)$ and mode of delivery ( $p 0.048$, OR 9.7) were also statistically significant.

\section{Discussion}

Cardiotoxic adverse effects of anthracyclines remain the major challenge for oncologist and cardiologist especially in developing countries where follow up compliance remains poor. Anthracycline associated adverse events include systolic and diastolic dysfunction, cardiomyopathy, arrhythmias and pericardial effusion. Patient can also present with asymptomatic cardiac dysfunction [1] [2] [4].

Table 2. Risk factors associated with anthracycline induced cardiac dysfunctions $(n=110)$.

\begin{tabular}{|c|c|c|c|c|c|}
\hline Demographic profile (n) & No dysfunction $(n=82)$ & Dysfunction $(n=28)$ & $p$-value & Crude odd ratio (CI) & Adjusted OR (CI) \\
\hline \multicolumn{6}{|l|}{ Age } \\
\hline$<5$ yrs $(46)$ & 37 (45\%) & $9(32 \%)$ & \multirow{2}{*}{0.276} & \multirow{2}{*}{$1.736(0.703-4.289)$} & \\
\hline$>5$ yrs (64) & $45(55 \%)$ & $19(68 \%)$ & & & \\
\hline \multicolumn{6}{|l|}{ Sex } \\
\hline Males (75) & $54(66 \%)$ & $21(75 \%)$ & \multirow{2}{*}{0.487} & \multirow{2}{*}{$1.556(0.590-4.102)$} & \\
\hline Females (35) & $28(34 \%)$ & $7(25 \%)$ & & & \\
\hline \multicolumn{6}{|l|}{ Type of malignancy } \\
\hline Hematological (94) & $71(87 \%)$ & $23(82 \%)$ & \multirow{2}{*}{0.756} & \multirow{2}{*}{$1.403(0.441-4.463)$} & \\
\hline Solid tumors (16) & $11(13 \%)$ & $5(18 \%)$ & & & \\
\hline Daunorubicin & $6(7 \%)$ & $10(36 \%)$ & \multirow{2}{*}{0.001} & \multirow{2}{*}{$7.037(2.262-21.895)$} & \multirow{2}{*}{-} \\
\hline Daunorubicin + Doxorubicin & $76(93 \%)$ & $18(64 \%)$ & & & \\
\hline \multicolumn{6}{|l|}{${ }^{*}$ Mode of delivery } \\
\hline Bolus (106) & $81(99 \%)$ & $25(89 \%)$ & \multirow{2}{*}{0.048} & \multirow{2}{*}{$9.72(0.968$ - 97.647) } & - \\
\hline Infusion (4) & $1(1 \%)$ & $3(11 \%)$ & & & \\
\hline \multicolumn{6}{|l|}{ Cumulative anthracycline dose } \\
\hline$<300 \mathrm{mg}(\mathbf{9 5})$ & 78 (95\%) & 17 (61\%) & \multirow{2}{*}{$<0.001$} & \multirow[t]{2}{*}{$12.618(3.583-44.43)$} & \multirow[t]{2}{*}{$2.25(0.032-0.522)$} \\
\hline >300 mg (15) & $4(5 \%)$ & $11(39 \%)$ & & & \\
\hline Radiation therapy (24) & $13(16 \%)$ & $11(39 \%)$ & 0.009 & $3.434(1.312-8.992)$ & $3.46(0.115-0.427)$ \\
\hline "Down’s syndrome (8) & $5(6 \%)$ & $3(11 \%)$ & 0.331 & $1.848(0.412-8.288)$ & \\
\hline Sepsis (47) & 28 (34\%) & $19(68 \%)$ & 0.002 & $4.071(1.631-10.166)$ & $2.58(0.040-0.305)$ \\
\hline
\end{tabular}

\footnotetext{
${ }^{*}$ As the sample size was less than 5 so fisher exact test was used.
} 
Recognition of dysfunction early, especially at the asymptomatic stage, significantly affects late outcome. In our study echocardiographic study showed 15 (13.6\%) developed cardiac dysfunction within a month and most of them (66\%) had isolated diastolic dysfunction. At 1 year, 28 (25\%) had dysfunction and most of 16/28 (57\%) had combined systolic and diastolic dysfunction. 18/110 (16\%) had signs and symptoms of congestive heart failure (CHF).

Several studies have reported up to 56\% subclinical echocardiographic cardiac dysfunction and incidence of clinical cardiotoxicity (CHF) ranged from 0 - 16\% [7]. Study conducted by Velensek et al. [18] showed isolated diastolic dysfunction in $28.6 \%$ of patients.

There is no safe dose limit and anthracycline can produce cardiotoxicity at any dose. But higher the cumulative dose more will be the cardiotoxicity and this is a well-recognized association. Hence this will always be considered a significant risk factor for cardiac damage and will also remain the best predictor for long term cardiotoxicity [19]. Van Dalen et al. [20] in his study showed that a cumulative dose of $300 \mathrm{mg} / \mathrm{m}^{2}$ or more was the only independent risk factor for anthracycline cardiotoxicity. Our study supports their findings as well as those suggested by other reports [19]-[21].

In our study AML and Ewing sarcoma had high tendency to develop cardiac dysfunction in acute and early onset phase. This was most likely due to their high anthracycline cumulative dose and primary disease. However there is no statistical significant anthracycline induced cardiotoxicity when hematological malignancies were compared with solid tumors $(p=0.756)$.

Doxorubicin and daunorubicin both are cardiotoxic. Doxorubicin, the parent drug, contributes the most to the cardiotoxicity, but for daunorubicin, the daunorubicinol metabolite contributes to about $75 \%$ of the cardiotoxicity. Conflicting data is present about anthracycline analogues and their cardiotoxicity differences [19] [22]. In our study whenever daunorubicin was used either alone or in combination with doxorubicin, the risk of cardiac dysfunction was significantly high $(p=0.001)$. Krischer et al. [8] in their study did not observe any difference for cardiotoxicity between doxorubicin and daunorubicin.

Godoy and associates [23] in their study showed that children younger than 4 years had a higher incidence of cardiotoxicity compared to those older than 4 years $(p<0.01)$. Other studies have suggested that females are at greater risk than males although the reason for this difference is not clear [2] [19]. In our study dysfunction was relatively common in younger children but neither age nor gender reach significance $(p=0.28$, and $p=0.49$ respectively) in relation to incidence of cardiac dysfunction that is comparable to other published studies [20]. Risk of cardiovascular disease increases with increasing radiation dose and time since therapy [24]. In our study $22 \%$ of patients received radiation therapy and in them risk for cardiotoxicity was high $(p=0.009)$.

Sepsis induced myocardial dysfunction is well known association; it potentiates anthracycline cardiotoxicity [19] [25]. Sepsis was a significant risk factor for cardiac dysfunction in our study $(p=0.002)$. The index of suspicion for subclinical cardiac failure should be high and ascertained by serial echocardiographic assessment particularly when high amount of fluid therapy is administered for sepsis and for tumor-lysis syndrome. This increase in preload can jeopardize cardiac function leading to frank congestive heart failure.

Increased cellular sensitivity to chemotherapy contributes to increased treatment-related morbidity and mortality for patients with DS with leukemia. Krischer et al. [8] found that trisomy 21 itself imparted a 3.4-fold relative risk for clinical cardiotoxicity [26]. We in our study did not find DS as a significant risk factor for cardiotoxicity $(p=0.331)$.

It is very well known that anthracycline induced cardiotoxicity is a slow progressive process that can occur even years after discontinuation of therapy [27]. Cardiac related mortality rate increases eight times and chances of developing cardiac dysfunction increases to 15 times more than the general population after 30 years of anthracycline treatment completion [28]. In our study one month's assessment showed that $14 \%$ had dysfunction which increased to $25 \%$ at 1 year after completion of therapy. At least a follow-up of six years is necessary to identify those long term survivors at risk for cardiac dysfunction, even asymptomatic patients, to prevent long term morbidity and mortality related to cardiovascular complications [29]. Unfortunately in developing countries like Pakistan we face significant morbidity and mortality due to multiple reasons like illiteracy, financial constraints and poor understanding of the need for follow-up. In our study 11 patients expired, out of them 7 had severe cardiac dysfunction.

\section{Conclusion}

Anthracyclines are important components of chemotherapy protocols but are associated with significant cardiac 
dysfunction. Cardiotoxicity is mostly related to high anthracycline cumulative dose, use of daunorubicin isolated or in combination with doxorubicin therapy, mode of delivery of anthracycline, concomitant radiation therapy and sepsis. Pericardial effusion occurs earlier and is an early marker for cardiotoxicity. Early diagnosis and prompt treatment of cardiac dysfunction may prevent significant mortality and morbidity. Regular long term follow-up with cardiologist is the key point for long term survival.

\section{Acknowledgements}

I acknowledge to Dr. Maha dev, Mr. Cyrus Tariq for their help in data collection.

\section{References}

[1] Singal, P.K. and Iliskovic, N. (1998) Doxorubicin-Induced Cardiomyopathy. The New England Journal of Medicine, 13, 900-905. http://dx.doi.org/10.1056/NEJM199809243391307

[2] Lipshultz, S.E., Alvarez, J.A. and Scully, R.E. (2008) Anthracycline Associated Cardiotoxicity in Survivors of Childhood Cancer. Heart, 4, 525-533.

[3] Roodpeyma, S., Moussavi, F. and Kamali, Z. (2008) Late Cardiotoxic Effects of Anthracycline Chemotherapy in Childhood Malignancies. Journal of Pakistan Medical Association, 12, 683-687.

[4] Monsuez, J.J., Charniot, J.C., Vignat, N. and Artigou, J.Y. (2010) Cardiac Side-Effects of Cancer Chemotherapy. International Journal of Cardiology, 144, 3-15. http://dx.doi.org/10.1016/j.ijcard.2010.03.003

[5] Cardinale, D., Sandri, M.T., Martinoni, A., Tricca, A., Civelli, M., Lamantia, G., Cinieri, S., Martinelli, G., Cipolla, C.M. and Fiorentini, C. (2000) Left Ventricular Dysfunction Predicted by Early Troponin I Release after High-Dose Chemotherapy. Journal of the American College of Cardiology, 36, 517-522. http://dx.doi.org/10.1016/S0735-1097(00)00748-8

[6] Puma, N., Ruggiero, A., Ridola, V., Maurizi, P., Lazzareschi, I., Attina, G., Mastrangelo, S., De Rosa, G. and Riccardi, R. (2008) Anthracycline-Related Cardiotoxicity: Risk Factors and Therapeutic Options in Childhood Cancers. Signa Vitae, 3, 30-34.

[7] Kremer, L.C., van Dalen, E.C., Offringa, M. and Voute, P.A. (2002) Frequency and Risk Factors of AnthracyclineInduced Clinical Heart Failure in Children: A Systematic Review. Annals of Surgical Oncology, 13, 503-512. http://dx.doi.org/10.1093/annonc/mdf118

[8] Krischer, J.P., Epstein, S., Cuthbertson, D.D., Goorin, A.M., Epstein, M.L. and Lipshultz, S.E. (1997) Clinical Cardiotoxicity Following Anthracycline Treatment for Childhood Cancer: The Pediatric Oncology Group Experience. Journal of Clinical Oncology, 4, 1544-1552.

[9] Tukenova, M., Diallo, I., Hawkins, M., Guibout, C., Quiniou, E., Pacquement, H., Dhermain, F., Shamsaldin, A., Oberlin, O. and de Vathaire, F. (2010) Long-Term Mortality from Second Malignant Neoplasms in 5-Year Survivors of Solid Childhood Tumors: Temporal Pattern of Risk According to Type of Treatment. Cancer Epidemiology, Biomarkers \& Prevention, 19, 707-715. http://dx.doi.org/10.1158/1055-9965.EPI-09-1156

[10] Von Hoff, D.D., Layard, M.W., Basa, P., Davis Jr., H.L., Von Hoff, A.L., Rozencweig, M. and Muggia, F.M. (1979) Risk Factors for Doxorubicin-Induced Congestive Heart Failure. Annals of Internal Medicine, 91, 710-717. http://dx.doi.org/10.7326/0003-4819-91-5-710

[11] Yeh, E.T. and Bickford, C.L. (2009) Cardiovascular Complications of Cancer Therapy: Incidence, Pathogenesis, Diagnosis, and Management. Journal of the American College of Cardiology, 53, 2231-2247. http://dx.doi.org/10.1016/j.jacc.2009.02.050

[12] Erdogan, D.Y.H., Youcel, H., Alanoglu, E.G., Uysal, B.A., Kocer, M., Ozaydin, M. and Dogan, A. (2011) Can Comprehensive Echocardiographic Evaluation Provide an Advantage to Predict Anthracycline-Induced Cardiomyopathy? Turk Kardiyoloji Dernegi Arsivi’s, 39, 646-653. http://dx.doi.org/10.5543/tkda.2011.01700

[13] Gatta, G., Capocaccia, R., Coleman, M.P., Ries, L.A. and Berrino, F. (2002) Childhood Cancer Survival in Europe and the United States. Cancer, 95, 1767-1772. http://dx.doi.org/10.1002/cncr.10833

[14] Sohn, D.W., Chai, I.H., Lee, D.J, Kim, H.C., Kim, H.S., Oh, B.H., Lee, M.M., Park, Y.B., Choi, Y.S., Seo, J.D. and Lee, Y.W. (1997) Assessment of Mitral Annulus Velocity by Doppler Tissue Imaging in the Evaluation of Left Ventricular Diastolic Function. Journal of the American College of Cardiology, 30, 474-480. http://dx.doi.org/10.1016/S0735-1097(97)88335-0

[15] Tei, C., Ling, L.H., Hodge, D.O., Bailey, K.R., Oh, J.K., Rodeheffer, R.J., Tajik, A.J. and Seward, J.B. (1995) New Index of Combined Systolic and Diastolic Myocardial Performance: A Simple and Reproducible Measure of Cardiac Function-A Study in Normals and Dilated Cardiomyopathy. International Journal of Cardiology, 26, 357-366. 
[16] Nagueh, S.F., Middleton, K.J., Kopelen, H.A., Zoghbi, W.A. and Quinones, M.A. (1997) Doppler Tissue Imaging: A Noninvasive Technique for Evaluation of Left Ventricular Relaxation and Estimation of Filling Pressures. Journal of the American College of Cardiology, 30, 1527-1533. http://dx.doi.org/10.1016/S0735-1097(97)00344-6

[17] Ommen, S.R., Nishimura, R.A., Appleton, C.P., Miller, F.A., Oh, J.K., Redfieldm M.M. and Tajik, A.J. (2000) Clinical Utility of Doppler Echocardiography and Tissue Doppler Imaging in the Estimation of Left Ventricular Filling Pressures: A Comparative Simultaneous Doppler-Catheterization Study. Circulation, 102, 1788-1794. http://dx.doi.org/10.1161/01.CIR.102.15.1788

[18] Velensek, V., Mazic, U., Krzisnik, C., Demsar, D., Jazbec, J. and Jereb, B. (2008) Cardiac Damage after Treatment of Childhood Cancer: A Long-Term Follow-Up. BMC Cancer, 8, 141. http://dx.doi.org/10.1186/1471-2407-8-141

[19] Barry, E., Alvarez, J.A., Scully, R.E., Miller, T.L. and Lipshultz, S.E. (2007) Anthracycline-Induced Cardiotoxicity: Course, Pathophysiology, Prevention and Management. Expert Opinion on Pharmacotherapy, 8, 1039-1058. http://dx.doi.org/10.1517/14656566.8.8.1039

[20] Van Dalen, E.C., van der Pal, H.J., Kok, W.E., Caron, H.N. and Kremer, L.C. (2006) Clinical Heart Failure in a Cohort of Children Treated with Anthracyclines: A Long-Term Follow-Up Study. European Journal of Cancer, 42, 31913198. http://dx.doi.org/10.1016/j.ejca.2006.08.005

[21] Nysom, K., Holm, K., Lipsitz, S.R., Mone, S.M., Colan, S.D., Orav, E.J., Sallan, S.E., Olsen, J.H., Hertz, H., Jacobsen, J.R. and Lipshultz, S.E. (1998) Relationship between Cumulative Anthracycline Dose and Late Cardiotoxicity in Childhood Acute Lymphoblastic Leukemia. Journal of Clinical Oncology, 16, 545-550.

[22] Gharib, M.I. and Burnett, A.K. (2000) Chemotherapy-Induced Cardiotoxicity: Current Practice and Prospects of Prophylaxis. European Journal of Heart Failure, 4, 235-242.

[23] Godoy, L.Y., Fukushige, J., Igarashi, H., Matsuzaki, A. and Ueda, K. (1997) Anthracycline-Induced Cardiotoxicity in Children with Malignancies. Acta Paediatrica Japonica, 39, 188-193. http://dx.doi.org/10.1111/j.1442-200X.1997.tb03579.x

[24] Lipshultz, S.E. and Adams, M.J. (2010) Cardiotoxicity after Childhood Cancer: Beginning with the End in Mind. Journal of Clinical Oncology, 28, 1276-1281. http://dx.doi.org/10.1200/JCO.2009.26.5751

[25] Court, O., Kumar, A. and Parrillo, J.E. (2002) Clinical Review: Myocardial Depression in Sepsis and Septic Shock. Critical Care, 6, 500-508. http://dx.doi.org/10.1186/cc1822

[26] Kowald, A. and Klipp, E. (2004) Alternative Pathways Might Mediate Toxicity of High Concentrations of Superoxide Dismutase. Annals of the New York Academy of Sciences, 1019, 370-374. http://dx.doi.org/10.1196/annals.1297.065

[27] Shankar, S.M., Marina, N., Hudson, M.M., Hodgson, D.C., Adams, M.J., Landier, W., Bhatia, S., Meeske, K., Chen, M.H., Kinahan, K.E., Steinberger, J. and Rosenthal, D. (2008) Monitoring for Cardiovascular Disease in Survivors of Childhood Cancer: Report from the Cardiovascular Disease Task Force of the Children's Oncology Group. Pediatric, 121, e387-396. http://dx.doi.org/10.1542/peds.2007-0575

[28] Lipshultz, S.E., Giantris, A.L., Lipsitz, S.R., Kimball Dalton, V., Asselin, B.L., Barr, R.D., Clavell, L.A., Hurwitz, C.A., Moghrabi, A., Samson, Y., Schorin, M.A., Gelber, R.D., Sallan, S.E. and Colan, S.D. (2002) Doxorubicin Administration by Continuous Infusion Is Not Cardioprotective: The Dana-Farber 91-01 Acute Lymphoblastic Leukemia Protocol. Journal of Clinical Oncology, 20, 1677-1682. http://dx.doi.org/10.1200/JCO.20.6.1677

[29] Lipshultz, S.E., Lipsitz, S.R., Sallan, S.E., Dalton, V.M., Mone, S.M., Gelber, R.D. and Colan, S.D. (2005) Chronic Progressive Cardiac Dysfunction Years after Doxorubicin Therapy for Childhood Acute Lymphoblastic Leukemia. Journal of Clinical Oncology, 23, 2629-2636. 
Scientific Research Publishing (SCIRP) is one of the largest Open Access journal publishers. It is currently publishing more than 200 open access, online, peer-reviewed journals covering a wide range of academic disciplines. SCIRP serves the worldwide academic communities and contributes to the progress and application of science with its publication.

Other selected journals from SCIRP are listed as below. Submit your manuscript to us via either submit@scirp.org or Online Submission Portal.
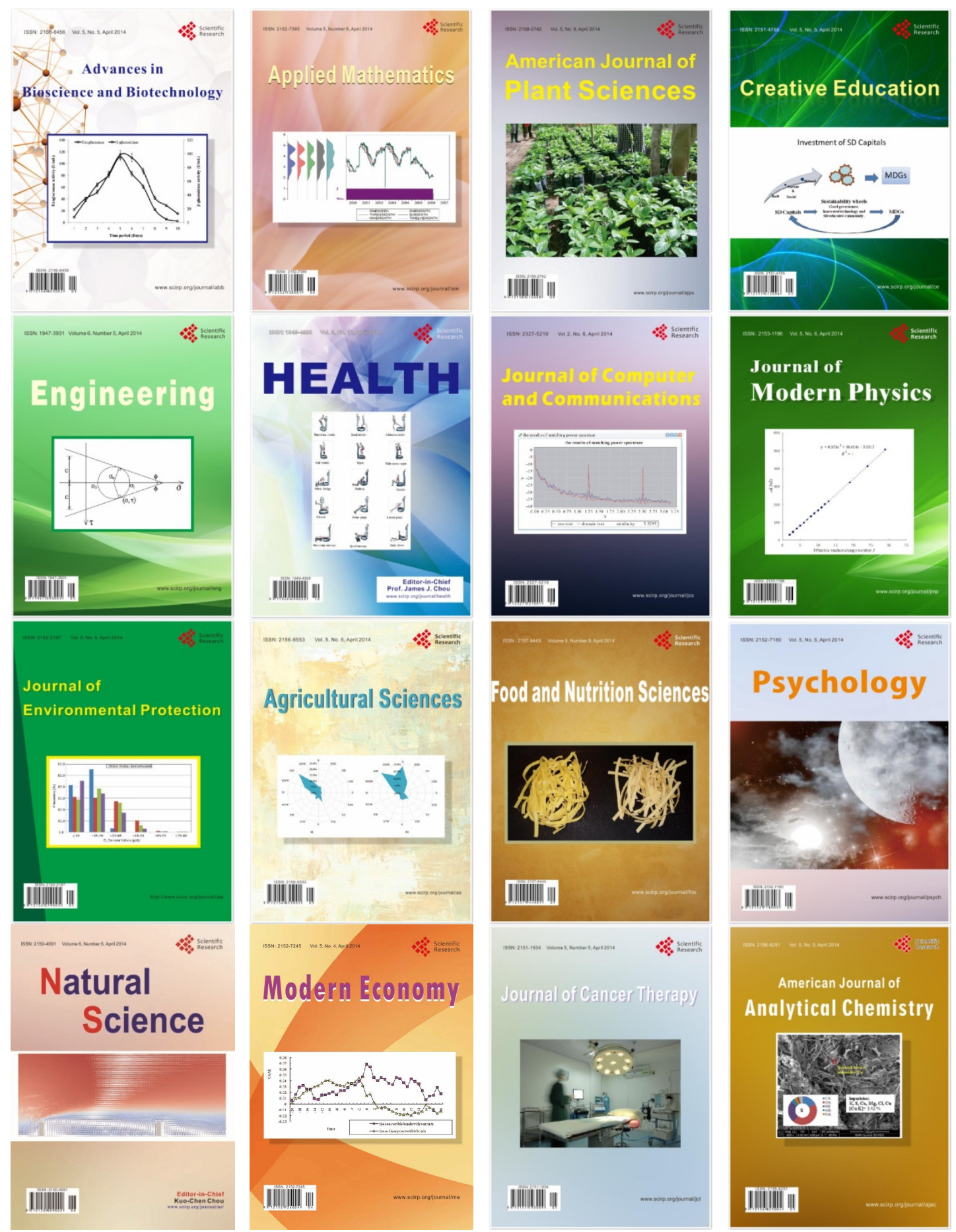